\title{
Element Level Identification of a Viscously Damped System
}

\author{
Subrata Chakraborty \\ Department of Civil Engineering, Bengal Engineering and Science University, Shibpur Howrah 711103 India
}

\author{
Sajal Roy \\ R\&DE (Engrs), DRDO, Dighi, Pune-15, India
}

(Received 22 November 2009; accepted 28 April 2010)

The modal data-based system identification (SI) algorithms normally consider the free vibration equation of motion of the dynamic system without damping for the identification of damage of existing structures. The present study intends to assess the damage of existing structures, including the effect of damping. The primary objective is to develop the equation error approach-based SI algorithm to identify both the stiffness properties and the damping parameters. The viscous damping model, which is computationally affordable and widely applicable to non-conservative systems, is considered for this objective. The formulation is hinged on the use of free vibration response in which the error norm of the eigen equation with damping is minimized. The SI algorithm is demonstrated through numerically-simulated modal data, which are obtained by the finite element analysis. The Monte Carlo simulation-based error sensitivity study confirms the consistency and robustness of the proposed algorithm.

\section{NOMENCLATURE}

\begin{tabular}{ll}
{$\left[k_{i}\right]$} & Element stiffness matrix \\
{$\left[m_{i}\right]$} & Element mass matrix \\
{$\left[c_{i}\right]$} & Element damping matrix \\
{$[M]$} & Global mass matrix \\
{$[K]$} & Global stiffness matrix \\
{$[C]$} & Global viscous damping matrix \\
$\{U\}$ & Global displacement vector \\
$\{\ddot{U}\}$ & Global acceleration vector \\
$\omega$ & Natural frequency \\
$\lambda$ & Eigenvalue \\
$\{\phi\}$ & Mode shape vector \\
$\varepsilon$ & Nonnegative error function \\
$\lambda_{k}$ & $k^{t h}$ eigenvalue \\
$\left\{\phi_{k}\right\}$ & $k^{t h}$ mode shape vector \\
${ }^{\prime} p^{\prime}$ & Total number of measured modes \\
$g_{i}, \delta_{i}$ & Damage parameter for $i^{t h}$ element \\
$\alpha_{i}, \beta_{i}$ & Damping parameters for $i^{t h}$ element \\
$\zeta_{1}, \zeta_{2}$ & Damping ratio of first two modes \\
{$\left[k_{u}\right]_{i}$} & Undamaged stiffness matrix for $i^{t h}$ element \\
{$\left[k_{d}\right]_{i}$} & Damaged stiffness matrix for $i^{t h}$ element \\
${ }^{\prime} n^{\prime}$ & Total number of elements in a system \\
${ }^{\prime} N^{\prime}$ & Total number of degrees of freedoms in a system \\
${ }^{\prime} n d e l^{\prime}$ & Total number of degrees of freedoms in an element \\
{$\left[L_{i}\right]$} & Matrix that links the local degrees of freedoms \\
& for $i^{t h}$ element to global degrees of freedoms \\
$\gamma$ & of the system \\
$\zeta_{i j}(k)$ & Relative magnitude of error \\
$\tilde{A}$ & Uniform random variate \\
$\mathrm{COV}$ & Coefficient of variation \\
\hline
\end{tabular}

\section{INTRODUCTION}

Structural Health Monitoring (SHM) is a real time structural condition assessment based on the feedback responses obtained directly from the structure. At its simplest level, the process involves knowing the occurrence of damage to determine the location, extent, and severity of damage. The problem becomes increasingly complex due to the existence of multiple damage locations in the structure, each of which may occur simultaneously or at different times. The interest in the ability to monitor a structure and detect the damage at the earliest possible stage is pervasive throughout the civil, mechanical, and aerospace engineering communities. To decide the remaining life and strengthening requirement for the safe performance of a structure, identification of the damage and determining the extent of this damage is important. The finite element (FE) model parameters, which are updated by SI techniques using modal data, have long been the topic in the field of damage detection of structures. These updating procedures can generally be categorized as either dynamic- or static- based techniques. The major development is focused on the dynamicbased techniques. In particular, the damage detection using modal data have been used extensively. A detailed review on the damage detection methods is provided by Doebling, et al., who cover various approaches of SI using static and dynamic data. ${ }^{1}$ Catbas and Aktan present various issues and promising indices for condition assessment and health monitoring, particularly for civil engineering structures. ${ }^{2}$ Chang, et al. review the health monitoring of civil infrastructure. ${ }^{3}$ The vibrationbased identification techniques are further reviewed in a number of publications. ${ }^{4-6}$ Alvandi and Cremona evaluate the reliability of various vibration-based SI techniques considering a simply supported beam with different damage levels using measured modal parameter. ${ }^{7}$ The SHM theory, technology, applications, critical new information on damage detection algorithms, novel sensor strategies and devices, networking, em- 